\title{
Lm-tLLO-neoantigens Vaccine ADXS-NEO
}

National Cancer Institute

\section{Source}

National Cancer Institute. Lm-tLLO-neoantigens Vaccine ADXS-NEO. NCI Thesaurus. Code 148380.

A proprietary, personalized plasmid DNA-based cancer vaccine composed of a liveattenuated strain of the Gram-positive bacterium Listeria monocytogenes (Lm) carrying a proprietary plasmid vector encoding multiple, patient-specific, immunogenic neoepitopes fused to a truncated form of the immunostimulant listeriolysin O (tLLO), with potential immunostimulatory and antineoplastic activities. Upon administration of the Lm-tLLO-neoantigens vaccine ADXS-NEO, the ADXS-NEO is taken up by antigen presenting cells (APCs), such as dendritic cells (DCs), and the expressed tLLO-neoantigens fusion protein is processed and presented to the immune system by both major histocompatibility complex (MHC) I and II molecules. This activates the immune system to exert both innate and adaptive immune responses involving the recruitment and activation of T-lymphocytes against the tumor-associated antigens (TAAs) specifically expressed by the patient's tumor cells, and inhibits the immunosuppressive tumorinfiltrating T-regulatory cells (Tregs) and myeloid-derived suppressor cells (MDSCs) within the tumor microenvironment (TME). This results in tumor cell lysis. The various unique mutation-derived TAAs that are used in ADXS-NEO are identified from a biopsy of the patient's tumor. 